\title{
EQUIVALENNCIA DE ESTÍMULOS EM PARTICIPANTES COM SÍNDROME DE DOWN: EFEITOS DA UTILIZAÇÃO DE PALAVRAS COM DIFERENÇAS MÚLTIPLAS OU CRÍTICAS E ANÁLISE DE CONTROLE RESTRITO DE ESTÍMULOS ${ }^{I}$
}

\author{
EQUIVALENCE RELATIONS IN CHILDREN WITH INTELLECTUAL DISABILITIES: \\ SEARCH ABOUT PROCEDURES USING STIMULI WITH MULTIPLE OR CRITICAL \\ DIFFERENCES AND RESTRICTED STIMULUS CONTROL
}

\author{
Camila Domeniconi e Júlio C. de Rose \\ UNIVERSIDADE FEDERAL DE SÃO CARLOS, BRASIL
}

\author{
EDSON M. HUZIWARA \\ UNIVERSIDADE DE SÃO PAULO, BRASIL
}

\begin{abstract}
RESUMO
O presente trabalho teve o propósito de investigar a formação de equivalência de estímulos com indivíduos portadores de Síndrome de Down. Foram ensinadas discriminaçôes condicionais auditivo-visuais para tais participantes, utilizando pseudo-palavras e manipulando distintamente o número de elementos idênticos (letras) presentes nas palavras em duas condiçōes experimentais. Os estímulos utilizados foram palavras dissílabas do tipo consoante mais vogal. Na primeira das condições experimentais, as palavras apresentavam diferenças múltiplas (uma ou duas letras em comum) e na segunda, diferenças críticas entre si (palavras com três ou quatro letras em comum). Os participantes foram quatro indivíduos com Síndrome de Down. Foram treinadas, por meio de procedimentos de emparelhamento com o modelo, as relações entre palavras ditadas e figuras, e entre palavras ditadas e impressas. Com dois participantes, treinou-se também as respostas de construção por meio da seleção ordenada de cada elemento do estímulo impresso. Foram testadas as nomeaçôes de palavras impressas e figuras e os emparelhamentos entre palavra impressa-figura e figura-palavra impressa. Os resultados sugerem que três dos quatro participantes apresentaram a formação de classes de equivalência nas duas condiçóes experimentais. O outro participante mostrou indícios de formação de classes apenas na primeira condição experimental. Dois participantes apresentaram maiores dificuldades nos treinos e testes da segunda condição. As dificuldades encontradas por eles podem ser atribuídas a controle restrito de estímulos.
\end{abstract}

Palavras-chave: equivalência de estímulos, leitura, similaridade entre os estímulos, controle de estímulos, Síndrome de Down

\begin{abstract}
The present study attempted to investigate stimulus equivalence formation by participants with Down's syndrome. Participants learned auditory-visual conditional discriminations with pseudo-words and was manipulated the stimulus similarity in two experimental conditions. The stimuli had multiple differences between them in the first condition, and had critical differences between them in the second condition. Four individuals with Down's syndrome participated. Relations between dictated words and pictures and between dictated and printed words were trained through matching to sample procedures. Two participants also learned responses of constructing the words by the ordinal selection of their elements. Performances tested were naming of printed words and pictures, and matching printed words to pictures and pictures to printed words. Results suggested that three of the four participants formed equivalence classes in both experimental conditions, whereas the other participant showed signs of class formation only in the first experimental condition. Two participants had more difficulties with training and testing in the second condition. The difficulties were attributed to restricted stimulus control.

Key words: stimulus equivalence, reading, stimulus similarity, stimulus control, Down's syndrome

\footnotetext{
1 Esta pesquisa contou com apoio financeiro do PRONEX/FAPESP (Processo número 03/09928-4), com bolsa de mestrado FAPESP para C.D. e bolsa de Produtividade em Pesquisa do CNPq para J.C.R. Os autores agradecem a revisão cuidadosa e as sugestōes de dois revisores anônimos que contribuíram para o aprimoramento do texto. Endereço para correspondência: Camila Domeniconi, Laboratório de Estudos do Comportamento Humano, UFSCar, Rodovia Washington Luís, 235. CEP 13565-905, São Carlos, SP. Email: camilad_psicologia@yahoo.com.br
} 
A equivalência de estímulos tem sido concebida como um modelo comportamental de função simbólica (de Rose, 1993; Sidman, 1994; Wilkinson \& McIlvane, 2001;) e sua ocorrência extensivamente documentada e replicada com populações de crianças e adultos com desenvolvimento típico e também com déficits de desenvolvimento (e.g., Carr, Wilkinson, Blackman \& McIlvane, 2000; Lazar, Davis-Lang \& Sanchez, 1984; Saunders, Wachter \& Spradlin, 1988; Sidman \& Tailby, 1982).

A habilidade de formar classes de estímulos equivalentes baseadas em relações arbitrárias entre os estímulos é fundamental para o uso da linguagem funcional, para a leitura com compreensão, dentre muitos outros comportamentos descritos como simbólicos (Mackay \& Sidman, 1984; Sidman, 1971, 1994; Spradlin \& Saunders, 1984).

O procedimento de escolha de acordo com o modelo tem sido utilizado para ensinar discriminações condicionais já que ele permite o estabelecimento de relações arbitrárias, potencialmente simbólicas entre estímulos fisicamente diferentes. Neste procedimento, o estímulo modelo controla qual o estímulo de comparação funciona como correto $\left(\mathrm{S}_{+}\right)$e qual (is) funcionam como estímulo incorreto (S-). Respostas ao estímulo de comparação determinado experimentalmente para ser correto, na presença do estímulo modelo específico, em geral são seguidas de reforçamento. Respostas aos estímulos definidos como incorretos, na presença daquele determinado modelo, não são seguidas de reforçamento (e.g., Barros, 1998; Green \& Saunders, 1998; Mackay, 1985).

Desta maneira, os treinos permitem o estabelecimento de discriminaçôes condicionais entre os estímulos modelo e os estímulos de comparação: um estímulo de comparação pode controlar uma resposta específica, a depender de um contexto específico que é determinado pela apresentação do estímulo modelo (Debert, Matos \& Andery, 2006). Originalmente abordado nos experimentos de Lashley (1938), o estabelecimento de tais relações vem recebendo destaque nos estudos sobre comportamento simbólico.

O treino pode ser realizado, por exemplo, com elementos de três conjuntos hipotéticos $\mathrm{A}$ $\left(A_{1}, A_{2}\right.$ e $\left.A_{3}\right), B\left(B_{1}, B_{2}\right.$ e $\left.B_{3}\right)$ e $C\left(C_{1}, C_{2}\right.$ e $\left.C_{3}\right)$. No ensino das relaçôes $A B$, em cada tentativa um estímulo do conjunto $A$ é apresentado como modelo, e os estímulos do conjunto B são apresentados simultaneamente, como estímulos de comparação. Nesse caso, os participantes devem selecionar o estímulo de comparação $\mathrm{B}_{1}$ diante do modelo $A_{1}$, não selecionando os estímulos $B_{2}$ e $B_{3}$. A escolha de $B_{1}$ produz reforço, enquanto que as escolhas de $B_{2}$ ou $B_{3}$ não possuem conseqüências programadas ou são seguidas por conseqüências não reforçadoras convencionadas para erros. De maneira similar, o reforço é contingente à escolha de $\mathrm{B}_{2}$ (e rejeição de $\mathrm{B}_{1}$ e $\mathrm{B}_{3}$ ) diante de $\mathrm{A}_{2}$, e à escolha de $\mathrm{B}_{3}$ (e rejeição de $\mathrm{B}_{1}$ e $\mathrm{B}_{2}$ ), diante de $\mathrm{A}_{3}$. No ensino das discriminações condicionais entre os elementos dos conjuntos A e $\mathrm{C}$, diante de um estímulo modelo $A_{1}$, a resposta a ser diferencialmente consequenciada será a escolha do estímulo $\mathrm{C}_{1}$ ao invés de $\mathrm{C}_{2}$ e $\mathrm{C}_{3}$; do mesmo modo, as escolhas de $\mathrm{C}_{2}$ e $\mathrm{C}_{3}$ diante de $\mathrm{A}_{2}$ e $\mathrm{A}_{3}$, respectivamente, também serão reforçadas. Estes treinos estabelecem relações funcionais entre os elementos estímulos dos conjuntos $A$, $B$ e $C\left(A_{1} B_{1}, A_{2} B_{2}, A_{3} B_{3} ; A_{1} C_{1}, A_{2} C_{2}, A_{3} C_{3}\right)$.

Contudo, nem todas as discriminações condicionais constituem relações de equivalência. Relações de equivalência são 
definidas por apresentarem as propriedades relacionais de reflexividade, simetria e transitividade (Sidman, 1994; Sidman \& Tailby, 1982). A reflexividade implica em que a relação de um elemento consigo próprio seja verdadeira, ou seja, $a \mathrm{r} a$ (onde $a$ pode ser qualquer elemento de um conjunto sob consideração e $\mathrm{r}$ a relação entre ambos). A simetria é constatada quando, tomados quaisquer dois elementos de um conjunto, a validade de $a$ r $b$ deve implicar, necessariamente, na validade da relação $b \mathrm{r} a$. Finalmente, se a validade das relações $a \mathrm{r} b$ e $b \mathrm{r} c$ implicar na validade da relação $a \mathrm{r} c$, então estará comprovada a transitividade (de Rose, 1993; Saunders \& Green, 1992; Sidman \& Tailby, 1982).

Diversos estudos da área têm replicado resultados positivos de formação de classes de equivalência após treinos de discriminação condicional com indivíduos com desenvolvimento típico. Contudo, indivíduos com atraso no desenvolvimento podem necessitar de maior quantidade de treino ou mesmo de procedimentos adicionais, tanto para a aquisição das discriminações condicionais treinadas quanto para a demonstração de relaçôes emergentes nos testes (e.g., Devany, Hayes \& Nelson, 1986; Eikeseth \& Smith, 1992). Essa maior dificuldade na aquisição de resultados positivos por indivíduos com atraso no desenvolvimento ficou clara nos resultados de Devany et al. (1986), que compararam a formação de equivalência em um grupo de crianças com desenvolvimento típico, um grupo de crianças com atraso no desenvolvimento e com repertório de linguagem e um outro grupo de crianças com atraso mas que não possuíam repertório de linguagem. Os resultados de Devany et al. (1986) mostraram que as crianças com desenvolvimento típico aprenderam as discriminações condicionais treinadas e formaram classes de equivalência. As crianças com atraso no desenvolvimento e com repertório de linguagem necessitaram de mais treino para aprender as discriminaçôes condicionais treinadas, mas também mostraram formação de classes de equivalência. As crianças que não possuíam repertório de linguagem, contudo, também necessitaram de mais treino para aprender as discriminações condicionais treinadas e não mostraram formação de equivalência, pelo menos na única sessão de teste que foi realizada neste experimento. Esses resultados levaram os autores a sugerir que a presença do repertório de linguagem poderia ser condição necessária para a formação de classes de equivalência.

É possível sugerir que fatores não relacionados à presença ou ausência de repertório explícito de linguagem possam explicar os resultados obtidos por Devany et al. (1986). O controle exercido por aspectos irrelevantes como a posição em que os estímulos eram apresentados, a preferência por estímulos específicos em função de história anterior ou o grau de similaridade física entre os estímulos são todos fatores que poderiam ser influenciado no desempenho dos participantes. Ainda, respostas podem ser dadas sob controle de menos aspectos do estímulo do que seriam necessários para obtenção de respostas acuradas, gerando um padrão de controle sob aspectos restritos dos estímulos. Este tipo de controle tem sido documentado em crianças com autismo ou atrasos no desenvolvimento (Allen \& Fuqua, 1985; Domeniconi, Costa \& de Rose, 2001; Lovaas, Koegel \& Schreibman, 1979; Lovaas, Schreibman, Koegel \& Rehm, 1971; Stromer, Mcllvane, Dube, \& Mackay, 1993). 
Birnie-Selwyn e Guerin (1997) manipularam a similaridade entre as palavras utilizadas em treinos de discriminação condicional auditivo-visual e testes de construção de palavras, a fim de verificar diferenças nos desempenhos de crianças com desenvolvimento típico em função desta manipulação. Foram utilizadas 24 palavras como estímulos modelo, todas elas eram iniciadas por encontros consonantais (palavras como snow, frog, grow, etc). Já os estímulos de comparação foram arranjados em dois grupos diferentes de palavras, em função dos mesmos modelos: em um dos grupos (grupo de estímulos com diferenças críticas), as palavras utilizadas como comparaçóes continham uma ou, no máximo, duas letras diferentes do modelo (no exemplo do modelo snow, as comparações foram slow e snap); no segundo grupo (estímulos com diferenças múltiplas), as palavras utilizadas como comparaçōes continham, três ou quatro letras diferentes do modelo (por exemplo, com o mesmo modelo, snow, as comparações utilizadas foram nice e rest). Os dados obtidos por eles apontaram que as crianças tiveram mais facilidade em emparelhar palavras diferentes (inseridas no treino com diferenças múltiplas) que palavras parecidas (treino com diferenças críticas). Em compensação, elas selecionaram com mais acurácia as letras constituintes daquelas palavras que foram treinadas em uma condição de diferenças críticas. Os autores discutiram os resultados destacando uma possível ocorrência de respostas dadas sob controle apenas da primeira letra de cada palavra, o que tornaria executável a tarefa de emparelhamento com o modelo, mas não a de seleção das letras constituintes da palavra, no caso da condição com diferenças múltiplas. Já o emparelhamento com o modelo na condição com diferenças críticas exigiria um olhar para a palavra toda, o que ensinaria este tipo de controle, facilitando a posterior resposta de construção dessas palavras. Portanto, a variável manipulada no presente estudo, ou seja, a similaridade entre os estímulos estaria relacionada com controle por partes ou elementos de estímulos compostos, o que tem sido denominado de controle restrito de estímulos.

O presente estudo teve o objetivo de ensinar discriminaçôes condicionais auditivovisuais para participantes com Síndrome de Down, utilizando pseudo-palavras e verificar a emergência de classes equivalentes. Além disso, foram utilizados dois grupos de palavras a fim de verificar os possíveis efeitos do uso de palavras com diferenças múltiplas ou críticas sobre os desempenhos individuais nos treinos e testes. Tal objetivo pauta-se na importância de se investigar como o treino e a emergência de discriminações condicionais podem envolver uma multiplicidade de relações e controles entre os estímulos. O conhecimento de algumas destas variáveis pode permitir adaptações necessárias para o treino de habilidades relevantes aos indivíduos com atraso no desenvolvimento cognitivo, como é o caso dos participantes do presente estudo.

\section{MÉTodo}

\section{Participantes}

Participaram do estudo dois adultos e duas crianças portadores de Síndrome de Down. A aplicação do teste Peabody Picture Vocabulary Test-revised (Dunn \& Dunn, 1981) permitiu a mensuração da idade mental do participante através da avaliação do seu nível de vocabulário receptivo. Informações detalhadas sobre os participantes estão apresentadas na Tabela 1. 
A participação no presente estudo ocorreu mediante consentimento prévio dos pais, atestado pela assinatura do Termo de Consentimento Livre e Esclarecido, submetido e aprovado pelo Comitê de Ética e Pesquisa da instituição.

\section{Local, equipamentos e materiais}

As sessões foram realizadas em uma sala do Laboratório de Estudos do Comportamento Humano da Universidade Federal de São Carlos, especialmente designada para esta finalidade, não sujeita a interrupção ou barulhos externos.

Foram utilizados um computador IBM $^{\circledR}$ com plataforma Windows ${ }^{\circledR}$ e monitor sensível ao toque, fones de ouvido, uma câmera filmadora, e o software "Lendo e Escrevendo em Pequenos Passos" (Rosa Filho, de Souza, de Rose \& Hanna, 1999). Além disso, foram utilizados materiais escolares e pequenos brinquedos, dispostos em prateleiras de um armário de madeira, simulando uma pequena "lojinha".

\section{Procedimento}

As sessões ocorreram uma vez por dia, quatro ou cinco vezes por semana, com duração média de vinte minutos.
Foram utilizados como estímulos palavras ditadas, figuras e palavras impressas (todas com quatro letras, sendo duas sílabas simples, do tipo consoante mais vogal). $\mathrm{O}$ procedimento de ensino foi dividido em duas condiçôes experimentais. Entre as condiçôes foi manipulada a similaridade entre as palavras impressas. Durante a Condição de Diferenças Múltiplas (DM) foram utilizadas palavras que apresentavam diferenças múltiplas entre si, ou seja, palavras com uma ou duas letras em comum (neste caso, pelo menos uma das letras em comum ocupava posiçôes diferentes em cada palavra). Na Condição de Diferenças Críticas (DC) foram utilizadas palavras que apresentavam diferenças críticas, ou seja, duas ou três letras em comum (com, pelo menos duas delas ocupando a mesma posição em cada palavra). As palavras utilizadas estão apresentadas na Tabela 2 .

Todos os participantes realizaram primeiro as atividades da Condição Experimental com Diferenças Múltiplas (DM) e depois as da Condição de Diferenças Críticas (DC).

As duas condiçôes experimentais foram planejadas para serem idênticas em relação aos procedimentos, atividades e conseqüências utilizadas, com diferenças apenas nos estímulos

Tabela 1

Caracterização dos participantes do estudo quanto à idade cronológica, idade equivalente ao vocabulário, sexo e alfabetização.

\begin{tabular}{ccccc}
\hline $\begin{array}{c}\text { Participantes } \\
\text { (nome fictício) }\end{array}$ & $\begin{array}{c}\text { Idade } \\
\text { cronológica }\end{array}$ & $\begin{array}{c}\text { Idade equivalente } \\
\text { ao vocabulário* }\end{array}$ & Sexo & Alfabetização \\
\hline Selma & $20 \mathrm{a} 6 \mathrm{~m}$ & $5 \mathrm{a} 2 \mathrm{~m}$ & $\mathrm{~F}$ & Sim \\
Paula & $24 \mathrm{a} 3 \mathrm{~m}$ & $5 \mathrm{a} 7 \mathrm{~m}$ & $\mathrm{~F}$ & Sim \\
Leandra & $7 \mathrm{a} 6 \mathrm{~m}$ & $2 \mathrm{a} 1 \mathrm{~m}$ & $\mathrm{~F}$ & Não \\
Luciano & $8 \mathrm{a} 10 \mathrm{~m}$ & $4 \mathrm{a} 10 \mathrm{~m}$ & $\mathrm{M}$ & Não \\
\hline
\end{tabular}

\footnotetext{
* avaliada através da aplicação do Peabody Picture Vocabulary Test
} 
Tabela 2

Palavras utilizadas em cada condição experimental.

\begin{tabular}{lcc}
\hline Condição Experimental & \multicolumn{2}{c}{ Palavras } \\
\hline \multirow{2}{*}{ Diferenças múltiplas } & Keni & Sota \\
& Xule & Bizu \\
& Ragi & Pafo \\
\hline \multirow{2}{*}{ Diferenças críticas } & Mado & Damo \\
& Mida & Dima \\
& Modi & Domi \\
\hline
\end{tabular}

utilizados, mas algumas alterações foram necessárias em função das dificuldades encontradas por dois participantes, em especial na Condição de Diferenças Críticas (DC), e serão detalhadas abaixo no item: "Procedimento de correção utilizado com Luciano e Leandra”.

Em cada uma das condições experimentais foram planejadas as seguintes atividades: pré-testes, treinos de emparelhamento palavra ditada-figura, testes de nomeação das figuras, treinos de emparelhamento palavra ditada-palavra impressa, testes de nomeação das palavras e testes de equivalência entre palavras impressas e figuras e vice-versa.

Respostas corretas, tanto em treinos quanto em testes, foram seguidas por conseqüências programadas no computador (palmas, animações sonoras ou mensagens de parabenização) e pelo recebimento de fichas, que eram trocadas por materiais escolares ou brinquedos disponíveis na "lojinha". Respostas erradas ocorridas durante as tentativas de treino eram seguidas por mensagens de correção (“Tente outra vez" ou "Não, não é”) e nas tentativas de teste produziam apenas a mudança para uma nova tentativa. As seqüências de modelos, as posições dos estímulos corretos, a quantidade de vezes que os estímulos apareciam como comparações e o tipo de relação testada em cada tentativa, eram balanceados e dispostos semi-randomicamente.

\section{Condição Experimental com Diferenças Múltiplas (DM)}

Pré-testes. Foram realizados pré-testes das seguintes relações: 24 tentativas de emparelhamento figura-figura (relação BB), 12 tentativas de nomeação de palavras (CD), 24 de emparelhamento palavra ditada-palavra impressa (AC) e 24 tentativas de emparelhamentos figura-palavra impressa e palavra impressa-figura (BC/CB). Os pré-testes não tinham nenhum critério de acerto e tinham o objetivo de verificar o repertório inicial dos participantes nas relações citadas utilizando as pseudo-palavras e figuras do procedimento.

Treinos de emparelhamento palavra ditadafigura (relação $A B$ ) e testes de nomeação das figuras (BD). A sessão era iniciada com a apresentação de seis tentativas envolvendo a nomeação das figuras utilizadas no passo de ensino.

O treino da relação entre palavras ditadas e figuras foi realizado gradualmente, inserindose as figuras do procedimento uma a uma. As atividades de treino foram iniciadas com tentativas de emparelhamento utilizando estímulos definidos, ou seja, palavras já previamente relacionadas às respectivas figuras (por exemplo: bolo, uva, relógio, rato). Em seguida, foram inseridos os nomes e figuras indefinidos, ou seja, aqueles não previamente relacionados entre si, sendo treinada uma relação nome-figura de cada vez, de modo que o participante sempre poderia excluir as comparações que já conhecia e selecionar a comparação nova, ao ouvir o nome novo. Para que uma nova relação palavra ditada-figura fosse 
introduzida, o participante deveria exibir um desempenho estável nas relações palavra ditadafigura ensinadas até aquele momento. $\mathrm{O}$ critério para determinar a estabilidade no desempenho do participante era um índice de acertos superior a 90\% em um bloco contendo 24 tentativas. Os blocos poderiam ser repetidos até que este critério fosse alcançado, com o limite máximo de três repetições por dia de um mesmo bloco.

Esse procedimento foi construído com base nos estudos sobre o responder por exclusão que demonstraram o potencial tecnológico deste procedimento (Costa, McIlvane, Wilkinson, \& de Souza, 2001; Dixon, 1977; Ferrari, de Rose \& Mcllvane, 1993).

A sessão era finalizada com a reapresentação das seis tentativas envolvendo a nomeação das figuras utilizadas no passo de ensino.

O critério de aprendizagem final (uma vez inseridas todas as seis relações entre os nomes e figuras do procedimento) era de 100\% de acertos somente nas tentativas envolvendo o emparelhamento de palavras ditadas e figuras. Esse bloco final (assim como os blocos anteriores, que ainda não continham todas as seis relações entre figuras e palavras ditadas) era repetido até que o participante alcançasse o critério, com o limite máximo de três repetições por dia. As tentativas de nomeação não possuíam critério de aprendizagem, mas eram repetidas a depender do desempenho do participante na tarefa de emparelhamento, uma vez que estavam inseridas ao final das tentativas de emparelhamento.

Treinos e testes de emparelhamento palavra ditada-palavra impressa (AC) e testes de nomeação das palavras impressas (CD). Para o treino das seis relações entre palavra ditada-palavra impressa foram programados dois blocos consecutivos de treino. Sendo assim, foram treinadas três palavras no primeiro bloco e mais três palavras no bloco seguinte. No primeiro bloco de sessões foram ensinadas as palavras "xule", "ragi” e "sota”, e as palavras "bizu", "keni” e "pafo" foram treinadas em seguida.

No início de cada sessão eram apresentadas três tentativas de emparelhamento entre palavra ditada e palavra impressa e três tentativas de nomeação das palavras impressas que seriam apresentadas naquela sessão. Estas tentativas ratificavam o desconhecimento prévio das relações por parte dos participantes, além de fornecer uma medida de comparação com os escores obtidos ao final da mesma sessão de treino.

$\mathrm{O}$ treino propriamente dito era iniciado com seis tentativas em que somente o modelo A1 era apresentado e ocorria a inserção gradual dos estímulos de comparação. Dessa maneira, a primeira tentativa continha apenas uma comparação, a segunda apresentava duas comparações e a terceira, três comparações. Em seguida eram apresentadas oito tentativas nas quais estavam presentes os estímulos A1 ou A2 como modelos. Depois havia 12 tentativas envolvendo os estímulos A1, A2 e A3 e, finalmente, 15 tentativas envolvendo todos os estímulos dispostos em ordem quase randômica. Cada um dos blocos de treino possuía como critério de aprendizagem $100 \%$ de acertos. A não obtenção do critério implicava na aplicação do mesmo bloco de treino até o limite máximo de três repetições cada dia.

Cada sessão era encerrada com a reapresentação das tentativas de emparelhamento entre palavra ditada e palavra impressa e nomeação de palavras impressas. As tentativas de nomeação das palavras não possuíam critério de aprendizagem, era apenas registrado o número de palavras lidas pelo participante após a sessão. Como dito no caso 
do emparelhamento entre palavra ditada e figura, também aqui essas tentativas de nomeação de palavras eram repetidas a depender do desempenho do participante na tarefa de emparelhamento, uma vez que estavam inseridas ao final das tentativas de emparelhamento.

Pós-testes - Testes de nomeação das palavras impressas (CD) e de emparelhamento palavra ditada-palavra impressa $(A C)$. Todas as palavras utilizadas no procedimento foram apresentadas em um bloco de teste composto por seis tentativas de nomeação das palavras impressas e seis tentativas de emparelhamento palavra ditada-palavra impressa.

Testes de emparelhamento palavra impressafigura e figura-palavra impressa (BC/CB). Os testes eram constituídos por blocos de doze tentativas. Seis delas continham a figura como modelo e três palavras impressas como estímulos de comparação. Além disso, a mensagem de voz "aponte a palavra" (emparelhamento figura-palavra impressa) era apresentada. Nas outras seis tentativas aparecia a palavra impressa como modelo, três figuras como comparações e a mensagem: "Aponte a figura" (emparelhamento palavra impressafigura). Os estímulos utilizados foram palavras impressas e figuras utilizadas nos treinos descritos acima.

Os pós-testes das relações $\mathrm{AC}, \mathrm{BD}, \mathrm{CD}$ e $\mathrm{BC} / \mathrm{CB}$ foram realizados mais de uma vez para alguns participantes após a repetição do treino de relações pertinentes a estes desempenhos (por exemplo, no caso de um desempenho muito baixo em um teste de nomeação de palavras, CD, o participante foi exposto novamente ao treino de emparelhamento palavra ditada-palavra impressa, $\mathrm{AC}$, e ao teste CD). Detalhes sobre quais testes foram repetidos e para quais participantes encontram-se na sessão de resultados.

As palavras utilizadas nesta condição estão apresentadas na porção superior da Tabela 2.

\section{Condição Experimental com Diferenças Criticas (DC) \\ O treino envolvendo palavras com} diferenças críticas seguiu a mesma seqüência apresentada para a Condição Experimental com Diferenças Múltiplas (DM), mudando apenas os estímulos. As palavras utilizadas nesta condiçãao estão apresentadas na porção inferior da Tabela 2.

As palavras "mado", "mida" e "modi" foram treinadas no primeiro bloco de sessões e as palavras "damo", "dima" e "domi" foram treinadas no segundo bloco de sessões da mesma condição experimental.

Procedimento de correção utilizado com Luciano e Leandra

Construção dos estímulos impressos a partir da seleção ordenada de cada sílaba componente da palavra modelo. Após a terceira repetição do primeiro bloco de treino de emparelhamento entre palavra ditada e palavra impressa foram introduzidas 24 tentativas de construção de acordo com o modelo. Diante da palavra impressa, os participantes deveriam compor uma palavra idêntica utilizando sílabas impressas disponibilizadas na tela do computador. A requisição de "cópia" objetivava colocar o comportamento dos participantes sob controle das unidades menores componentes de cada palavra e melhorar os desempenhos nos treinos de emparelhamento palavra ditada palavra impressa e nos testes de nomeação de palavras. O critério de aprendizagem requerido era de $90 \%$ de acertos. Caso o critério não fosse satisfeito o 
treino deveria ser repetido até o limite máximo de três repetições por dia. Após a realização do treino de resposta construída os participantes foram novamente expostos aos treinos e testes de emparelhamento palavra ditada-palavra impressa, testes de nomeação de palavras e testes de emparelhamento figurapalavra impressa, palavra impressa-figura.

\section{Resultados}

A análise dos dados foi realizada de acordo com as porcentagens de acertos obtidas por cada participante em cada um dos testes e está representada na Figura 1.

A Figura 1 apresenta os resultados individuais de desempenho de cada participante nas seguintes relações testadas antes (pré-testes) e depois dos treinos (pós-testes): nomeação de figuras (BD), nomeação de palavras impressas (CD), emparelhamento palavra ditada-palavra impressa (AC) e emparelhamento figura-palavra impressa, palavra impressa-figura (BC/CB). Os resultados apresentados na Figura 1 referem-se sempre ao desempenho demonstrado pelo participante na última aplicação dos testes. No texto abaixo está especificado se o participante foi exposto ao pós-teste mais de uma vez.

As barras de cor cinza representam os resultados obtidos na Condição Experimental com Diferenças Múltiplas (DM) e as pretas, os resultados da Condição Experimental com Diferenças Críticas (DC).

\section{Condição Experimental com Diferenças Múltiplas}

Todos os resultados obtidos nos testes desta condição experimental estão descritos nas barras de cor cinza dos gráficos na Figura 1.

Pré-testes. Como pode ser observado nas barras de cor cinza à esquerda dos dois primeiros gráficos da Figura 1, as participantes Selma e Paula obtiveram 100\% de acertos em todas as tentativas do pré-teste com as pseudo-palavras, com exceção para as tentativas de nomeação de figuras (BD), em que ambas não obtiveram acertos, e para as tentativas de teste de emparelhamento palavra impressa-figura, figura-palavra impressa (BC/CB), nas quais a participante Selma acertou 65\% das tentativas e Paula acertou $30 \%$ das tentativas.

Os participantes Luciano e Leandra iniciaram o procedimento com porcentagens abaixo do nível do acaso em, praticamente, todas as atividades de pré-testes. Pode-se observar que nenhum dos dois obteve acertos na nomeação de figuras (BD), de palavras impressas (CD) e de emparelhamento palavra ditada-palavra impressa (AC). Porcentagens de acertos próximas do nível do acaso também foram observadas nas atividades de emparelhamento figura-palavra impressa, palavra impressa-figura (BC/CB). Leandra obteve 30\% de acerto e Luciano 25\%.

Os resultados apresentados à direita de cada gráfico de Figura 1 são relativos aos dados obtidos após a realização dos treinos (pós-testes).

Testes de nomeação das figuras (BD). Os participantes Selma, Leandra e Luciano obtiveram 100\% de acertos nos testes de nomeação das figuras na primeira exposição às atividades. Paula não nomeou corretamente nenhuma figura e passou novamente pelo treino de emparelhamento palavra ditada-figura e pelos testes de nomeação das figuras. Mesmo após a segunda exposição da participante aos treinos e testes ela nomeou apenas 20\% das figuras (o resultado representado na Figura 1 refere-se à segunda exposição).

Testes de nomeação das palavras impressas (CD). Selma e Paula nomearam todas as palavras 
Pré-testes

Pós-testes

Selma

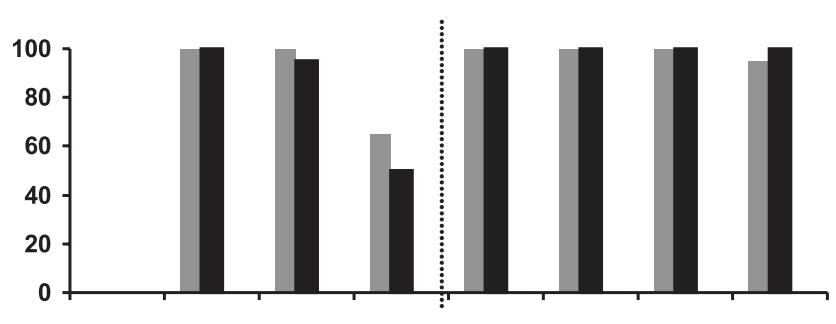

Paula
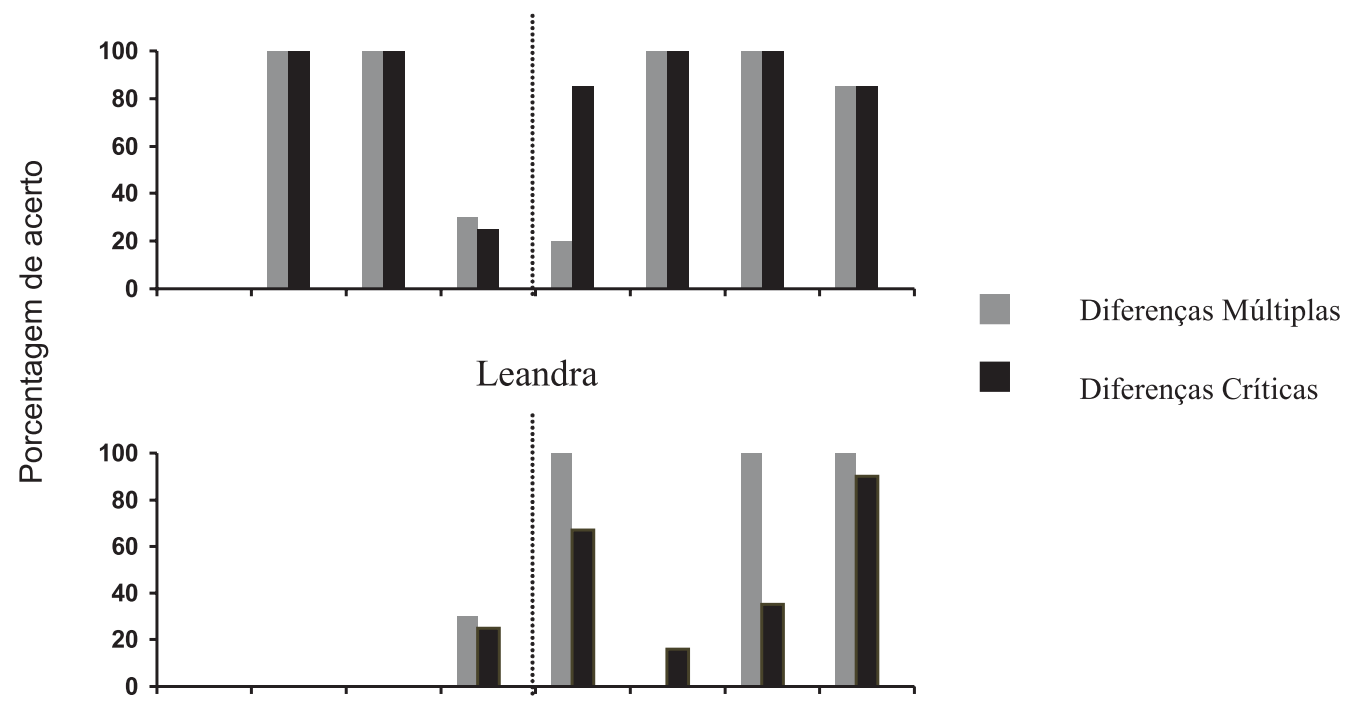

Luciano

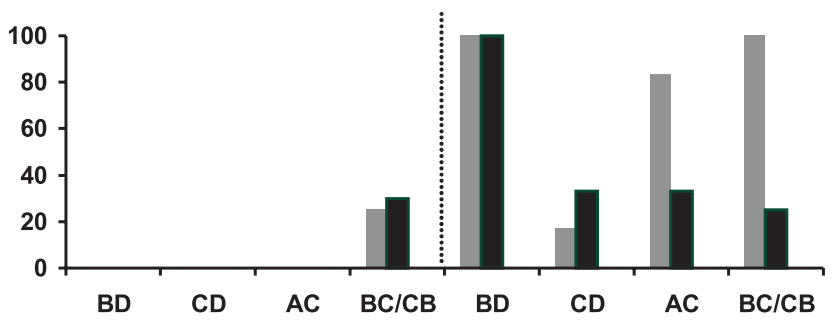

Relações testadas

Figura 2. Porcentagens de acertos em cada uma das relações testadas: nomeação de figuras (BD), nomeação de palavras (CD), emparelhamento palavra ditada-palavra impressa (AC), emparelhamento figura-palavra impressa, palavra impressafigura $(\mathrm{BC} / \mathrm{CB})$. As barras em cor cinza representam os resultados da Condição Experimental com Diferenças Múltiplas e as barras pretas representam os resultados da Condição Experimental com Diferenças Críticas. 
impressas, já Leandra não nomeou nenhuma palavra e Luciano apenas uma (16,7\% de acerto). Também com Luciano e Leandra tentou-se repetir uma vez todo o treino de emparelhamento palavra ditada-palavra impressa $(A C)$ e refazer o teste de nomeação das palavras (CD), a fim de verificar se esta nova exposição às contingências de treino e teste melhoraria o desempenho do participante. Esta melhora não ocorreu, como pode ser observado no resultado que se refere à segunda exposição, neste caso, idêntico ao obtido na primeira.

Testes de emparelhamento palavra ditadapalavra impressa (AC). As participantes Selma, Paula e Leandra realizaram corretamente 100\% das atividades de emparelhamento palavra ditada-palavra impressa. Luciano acertou 83,3\% das atividades. Os dados de Leandra e Luciano referem-se à segunda exposição aos testes. Os treinos e testes de emparelhamento AC foram repetidos com estes dois participantes na tentativa de melhorar os percentuais obtidos na nomeação das palavras impressas (descritos acima).

Testes de emparelhamento figura-palavra impressa e palavra impressa-figura (BC/CB). A participante Selma obteve $95 \%$ de acerto na segunda exposição aos testes de emparelhamento $\mathrm{BC} / \mathrm{CB}$. Os testes foram repetidos para verificar se ocorreria a emergência desta relação, uma vez que ela havia obtido escores excelentes nas outras medidas. Os resultados da participante Paula também se referem à segunda exposição ao teste, na qual ela obteve $83,3 \%$ de acertos. $\mathrm{Na}$ primeira vez em que realizaram os testes BC/CB, Selma e Paula obtiveram, respectivamente, 75 e $30 \%$ de acerto.

Leandra e Luciano obtiveram 100\% de acertos na primeira exposição aos testes $\mathrm{BC} / \mathrm{CB}$.

Em geral, observa-se através dos dados apresentados na Figura 1 que a Condição DM pareceu ser favorecedora, especialmente para os participantes Leandra e Luciano, para a aquisição de repertórios receptivos, como o emparelhamento entre palavras ditadas e palavras impressas (AC). Esta condição também foi favorável à formação de classes entre palavras impressas e figuras e vice versa $(\mathrm{BC} / \mathrm{CB})$ para o participante Luciano.

Já em relação ao repertório expressivo, a condição de treino não pareceu ser tão favorável, especialmente para Leandra e Luciano, que obtiveram resultados inferiores na nomeação de palavras (CD), quando comparados à próxima Condição Experimental, embora estas diferenças não tenham sido significativas.

\section{Condição Experimental com Diferenças Críticas}

Todos os resultados obtidos nos testes desta condição experimental estão descritos nas barras de cor preta dos gráficos na Figura 1.

Pré-testes. As participantes Selma e Paula apresentaram 100\% de acertos nas atividades de nomeação das palavras (CD) e de emparelhamento palavra ditada palavra impressa (AC). Novamente as porcentagens mais baixas de acerto puderam ser observadas na nomeação das figuras (nenhuma das participantes nomeou corretamente nenhuma figura) e nos emparelhamentos figura-palavra impressa palavra impressa-figura (BC/CB). Neste teste, Selma acertou 50\% das tentativas e Paula, 25\%.

Leandra e Luciano, a exemplo do que ocorrera na condição DM, não apresentaram acertos na nomeação de figuras (BD), de palavras impressas (CD) e de emparelhamento palavra ditada-palavra impressa (AC). Nas atividades de emparelhamento figura-palavra impressa, palavra impressa-figura (BC/CB), Leandra obteve 25\% de acerto e Luciano, 30\%. 
Novamente, as barras na cor cinza representam os dados obtidos após a realização dos treinos (pós-testes).

Testes de nomeação das figuras (BD). Selma obteve $100 \%$ acertos, Paula nomeou corretamente $85 \%$ das figuras, Leandra $67 \%$ e Luciano $100 \%$. Os dados se referem à primeira exposição aos testes.

Testes de nomeação das palavras impressas (CD). Selma e Paula nomearam corretamente todas as palavras do procedimento $(100 \%$ de acerto) na primeira exposição aos testes, Leandra nomeou apenas uma palavra ( $16,7 \%$ de acerto) e Luciano duas palavras (33,3\% de acerto). Os dados de Leandra e Luciano referem-se à segunda exposição aos testes, após três repetições de todo o treino de emparelhamento palavra ditadapalavra impressa (AC) e a inserção do procedimento de ensino baseado na construção do modelo impresso a partir de seleção ordenada das sílabas correspondentes. Na primeira exposição aos testes, os dois participantes obtiveram porcentagens nulas de acerto.

Testes de emparelhamento palavra ditadapalavra impressa (AC). Selma e Paula emparelharam corretamente $100 \%$ das palavras impressas aos respectivos modelos auditivos, na primeira exposição aos testes. Leandra e Luciano obtiveram 33,3\% de acerto após a terceira repetição dos treinos e testes de emparelhamento $\mathrm{AC}$ e da inserção do procedimento de ensino baseado na construção do modelo impresso a partir de seleção ordenada das sílabas correspondentes. $\mathrm{Na}$ primeira vez em que realizaram os testes $\mathrm{AC}$, Leandra e Luciano obtiveram, respectivamente, zero e $33,3 \%$ de acerto.

Testes de emparelhamento figura-palavra impressa palavra impressa-figura (BC/CB). Selma e Paula obtiveram respectivamente 100 e $85 \%$ de acerto nos testes BC/CB. Os dados de Selma referem-se à primeira exposição ao teste e os de Paula a segunda exposição (Paula obteve 30\% de acerto na primeira exposição). Leandra obteve $90 \%$ de acerto e Luciano $25 \%$ de acerto, na primeira exposição ao teste.

Pode-se observar em termos de tendência geral, a partir da observação dos dados apresentados na Figura 1, que a Condição Experimental com Diferenças Críticas (DC) constituiu uma situação de aprendizagem mais difícil, especialmente para os participantes Leandra e Luciano, que obtiveram consideravelmente menos acertos

Tabela 3

Número de sessões de treino para cada participante até a obtenção do critério de aprendizagem nas discriminações condicionais.

\begin{tabular}{|c|c|c|c|c|}
\hline \multirow[t]{2}{*}{ Participante } & \multicolumn{2}{|c|}{$\begin{array}{l}\text { Condição Experimental com } \\
\text { Diferenças Múltiplas (DM) }\end{array}$} & \multicolumn{2}{|c|}{$\begin{array}{c}\text { Condiçãao Experimental com } \\
\text { Diferenças Críticas (DC) }\end{array}$} \\
\hline & Treino AB & Treino AC & Treino AB & Treino AC \\
\hline Selma & 14 & 3 & 8 & 4 \\
\hline Paula & 18 & 5 & 6 & 4 \\
\hline Leandra & 22 & 26 & 10 & 39 \\
\hline Luciano & 8 & 20 & 8 & 32 \\
\hline
\end{tabular}


nas tarefas de emparelhamento palavra ditada palavra impressa (AC) e nos testes de equivalência entre palavras impressas e figuras e vice-versa (BC/CB), no caso de Luciano. Apesar destes dados nas tarefas receptivas, os dados de nomeação das palavras (CD) foram melhores nesta condição embora esta diferença não seja significativa.

A Tabela 3 apresenta o número de sessões de treino necessário para que cada um dos participantes atingisse o critério de aprendizagem. A análise dos dados apresentados nela nos permite fazer mais consideraçóes sobre o desempenho dos participantes em função das duas condições experimentais, especialmente sobre qual delas constituiu uma situação de aprendizagem de discriminações condicionais mais difícil.

\section{DisCUSSĀO}

Quatro participantes com Síndrome de Down realizaram treinos de discriminação condicional entre palavras ditadas e figuras (relação $\mathrm{AB}$ ) e entre palavras ditadas e impressas (AC). Além destes treinos, dois dos participantes realizaram treinos de construção das palavras a partir da seleção ordenada das sílabas componentes do modelo em uma das condições experimentais programadas. Finalmente, todos realizaram testes de nomeação das figuras e testes de equivalência entre figuras e palavras impressas e vice versa.

O procedimento incluiu a manipulação da similaridade entre as palavras, proposta no estudo de Birnie-Selwyn e Guerin (1997) e foram programadas duas condições experimentais: condição experimental com diferenças múltiplas (DM) e condição experimental com diferenças críticas (DC).
Todos os participantes realizaram primeiro as atividades da condição DM e depois da condição DC. Essa ordem foi definida com o propósito de proporcionar uma dificuldade crescente nas atividades realizadas pelos participantes, acreditando que pode ser mais simples realizar discriminaçôes condicionais quando os estímulos são facilmente discrimináveis (pouco similares) do que realizar discriminações condicionais com estímulos muito parecidos.

Alguns estudos trataram da similaridade física dos estímulos como uma variável importante na estimativa da dificuldade de discriminação (p.ex. Birnie-Selwin \& Guerin, 1997; Guttman \& Kalish, 1956). Considerando o grau de dificuldade de uma tarefa como uma característica que pode ser avaliada a partir de uma análise do desempenho dos indivíduos ao executá-la (Oliveira-Castro, Coelho \& Oliveira-Castro, 1999), os resultados obtidos por Luciano e Leandra indicaram que a escolha pela ordem das condições experimentais foi adequada. De fato, a ordem das condições experimentais proporcionou um grau crescente de dificuldade especialmente quando se observa os dados dos testes de emparelhamento palavra ditada e palavra impressa. Além disso, o número de sessões necessário para que cada participante atingisse o critério de aprendizagem para os treinos também parece sugerir a adequação na ordem de treino utilizada.

Os dados de Luciano e Leandra replicaram os obtidos por Birnie-Selwin e Guerin (1997) no que concerne às porcentagens de acerto nos emparelhamentos entre palavras ditadas e impressas (relação AC), comparando as duas condições experimentais. Observa-se na Figura 1 que ambos obtiveram escores 
significativamente melhores na condição experimental com diferenças múltiplas que na condição com diferenças críticas, mesmo com uma maior exposição aos treinos e testes da condição com diferenças críticas (os escores da condição com diferenças múltiplas são relativos à segunda exposição e os da condição com diferenças críticas, à terceira exposição).

Investigações sobre os processos envolvidos na aquisição das habilidades de ler, soletrar ou construir palavras têm chamado atenção para a necessidade de analisar detalhadamente o controle de estímulos envolvido em alguns erros típicos de leitura e soletração, tendo em vista a necessidade de controle por todos os elementos da palavra, para a obtenção de respostas acuradas (Dube, McDonald, Mcllvane \& Mackay, 1991; Stevens, Blackhurst \& Slaton, 1991) e, portanto, mais adaptadas. A interpretação dos dados de Luciano e Leandra permite explanações sobre o tipo de controle que atuou durante as respostas, uma vez que o desempenho de emparelhamento palavra ditada palavra impressa na condição com diferenças múltiplas foi muito superior ao desempenho com diferenças críticas. Pode-se supor que eles responderam às atividades de ambas as condiçôes sob controle de algum aspecto restrito da palavra, possivelmente a primeira letra, uma vez que este tipo de controle era possível nesta condição e compatível com a produção de respostas acuradas. $\mathrm{Na}$ segunda condição, respostas sob controle de aspectos restritos da palavra eram incompatíveis com a produção de respostas acuradas, uma vez que as palavras diferiam em uma ou mais letras. Evidência do tipo de controle que atuou sobre a emissão das respostas de escolha nos emparelhamentos palavra ditada palavra impressa é que os escores verificados na segunda condição experimental foram significativamente menores que os da primeira. Além do mais, o número de sessões de treino necessário para que esses participantes atingissem os critérios de acertos estipulados nas sessões de treino de emparelhamento palavra ditada-palavra impressa foi significativamente maior na segunda condição experimental, evidenciando a maior dificuldade encontrada por eles.

Tendo em vista as dificuldades encontradas por Luciano e Leandra na realização da discriminação entre palavras com diferenças críticas, além dos procedimentos descritos anteriormente, foi introduzido o procedimento de construção das palavras impressas a partir da seleção ordenada de cada sílaba componente da palavra modelo. Este procedimento foi utilizado por Dube et. al. (1991) e constitui um tipo de resposta diferencial descrito na literatura como um possível procedimento de remediação nos casos em que o desempenho do participante leve a suspeita de que ele não está respondendo sob controle de todos os elementos de um estímulo complexo. A tarefa de construção de uma palavra visa ensinar o participante a atentar para todos os elementos da mesma, uma vez que é muito difícil completar a tarefa sem tal discriminação.

A introdução do procedimento de construção das palavras não foi suficiente para que os dados dos dois participantes nas tarefas de discriminação auditivo visual melhorassem para além da porcentagem de acerto que pode ser considerada ao acaso (35\% de acerto Leandra e 33\% de acerto Luciano). Em relação a este aspecto, pode-se apontar a interferência da quantidade de erros cometidos pelos participantes até este ponto do procedimento como uma variável que pode ter contribuído para diminuir a eficácia do procedimento. 
Estudos mostram que, durante a aquisição de uma discriminação, um dos efeitos de um grande número de respostas ao estímulo incorreto (consideradas experimentalmente como erradas) diz respeito ao controle do comportamento do participante por aspectos irrelevantes, conduzindo a um possível desempenho persistentemente falho na discriminação presente e, talvez, até em outras relacionadas (Stoddard \& Sidman, 1967; Terrace, 1963a; Terrace, 1963b).

Apesar da grande quantidade de erros apontada no desempenho de dois participantes durante a condição com diferenças críticas, em relação aos dados de equivalência de estímulos, três dos quatro participantes mostraram indícios de estabelecimento de relações de equivalência entre palavras impressas e as respectivas figuras, nunca relacionadas durante os treinos, nas duas condições experimentais. O outro participante (Luciano) apresentou tais indícios apenas nos testes da condição com diferenças múltiplas, corroborando com os dados que atestam o potencial tecnológico do procedimento de emparelhamento com o modelo para a formação de classes de equivalência, mesmo quando o participante apresenta déficits de desenvolvimento e necessite de uma maior quantidade de treino, como no presente estudo.

Os resultados de nomeação das palavras replicaram os dados da literatura da área de equivalência de estímulos, que mostraram que o repertório receptivo instalado através dos treinos de emparelhamento com o modelo pode não garantir a nomeação dos estímulos envolvidos nos testes de equivalência, indicando que talvez sejam repertórios independentes (p.ex., Guess \& Baer, 1973; Lee, 1981; Lee \& Pegler, 1982; Mackay \& Sidman, 1984; Sidman, Willson-Morris \& Kirk, 1986). Em especial, observa-se que Leandra e Luciano nomearam, respectivamente, nenhuma e apenas uma palavra na Condição DM e obtiveram resultados de $100 \%$ de acerto nos testes de equivalência. Ainda na Condição DC, Leandra nomeou apenas uma palavra, mas obteve $95 \%$ de acerto nos testes de equivalência. Os resultados de Selma e Paula não permitiram essa comparação uma vez que elas já possuíam a habilidade de nomear as palavras antes dos treinos.

Pesquisas posteriores poderiam ser realizadas com foco na investigação de controle restrito de estímulos em participantes com atrasos de desenvolvimento, uma vez que o presente estudo soma-se aos estudos anteriores na sugestão de que este tipo de controle pode estar ocorrendo e dificultando a aquisição de relações baseadas em estímulos compostos, como é o caso das palavras (Allen \& Fuqua, 1985; Domeniconi et al., 2001; Litrownick, McInnis, Wetzel-Prichard \& Felipelli, 1978; Lovaas et. al., 1971; Lovaas et al., 1979; Stromer, McIlvane \& Dube, 1993).

\section{REFERÊNCIAS}

Allen, K. D., \& Fuqua, W. (1985). Eliminating selective stimulus control: a comparison of two procedures for teaching mentally retarded children to respond to compound stimuli. Journal of Experimental Child Psychology, 39, 55-71.

Barros, R. (1998). Controle do comportamento por relaçōes entre estímulos. Tese de doutorado não publicada, Instituto de Psicologia, Universidade de São Paulo, São Paulo, Brasil.

Birnie-Selwyn, B., \& Guerin, B. (1997). Teaching children to spell: decreasing consonant cluster errors by eliminating selective stimulus control. Journal of Applied Behavior Analysis, 30, 69-92. 
Carr, D., Wilkinson, K. M., Blackman, D., \& McIlvane, J. W. (2000). Equivalence classes in individuals with minimal verbal repertoires. Journal of the Experimental Analysis of Behavior, 74, 101-114.

Costa, A. R. A., Mcllvane, J. W., Wilkinson, K. M., \& de Souza, D. G. (2001). Emergent word-object mapping by children: further studies using the blank comparison technique. The Psychological Record, 51, 343-355.

Debert, P., Matos, M. A., \& Andery, M. A. P. A. (2006). Discriminação condicional: definições, procedimentos e dados recentes. Revista Brasileira de Análise do Comportamento, 2, 125-133.

de Rose, J. C. C. (1993). Classes de estímulos: implicações para uma análise comportamental da cognição. Psicologia: Teoria e Pesquisa, 9, 283-303.

Devany, J. N., Hayes, S. C., \& Nelson R. O. (1986). Equivalence class formation in language-able and languange-disabled children. Journal of the Experimental Analysis of Behavior, 46, 243-457.

Dixon, L. S. (1977). The nature of control by spoken words over visual stimulus selection. Journal of Experimental Analysis of Behavior, 27, 433-442.

Domeniconi, C., Costa, A. R. A., \& de Rose, J. C. C. (2001). Controle restrito de estímulos em crianças com desenvolvimento normal e individuos com Síndrome de Down. Anais do X Encontro Brasileiro de Psicoterapia e Medicina Comportamental, Campinas-SP, CD-Room.

Dube, W. V., McDonald, S. J., McIlvane, W. J., \& Mackay, H. A. (1991). Constructed-response matching to sample and spelling instruction. Journal of Applied Behavior Analysis, 24, 305-317.

Dunn, L. M., \& Dunn, L. M. (1981). Peabody Picture Vocabulary Test-Revised. Circle Pines, MN: American Guidance Service.

Eikeseth, S., \& Smith, T. (1992). The development of functional and equivalence classes in high-functioning autistic children: The role of naming. Journal of the Experimental Analysis of Behavior, 58, 123-133.
Ferrari, C., de Rose, J. C. C., \& McIlvane, W. J. (1993). Exclusion vs. selection training of auditory-visual conditional relations. Journal of Experimental Child Psychology, 56, 49-63.

Green, G., \& Saunders, R. R. (1998). Stimulus equivalence. In: A. Lattal \& M. Perone (Eds.). Handbook of Research Methods in Operant Behavior. pp. (229-261). Nova York: Plenum Press.

Guess, D., \& Baer, D. M. (1973). Analysis of individual differences in generalization between receptive and productive language in retarded-children. Journal of Applied Behavior Analysis, 6, 311-329.

Guttman, N., \& Kalish, H. I. (1956). Discriminability and stimulus generalizacion. Journal of Experimental Psychology, 51, 79-88.

Lashley, K. S. (1938). Conditional reactions in the rat. Journal of Psychology, 6, 311-324.

Lee, V. L. (1981). Prepositional phrases spoken and heard. Journal of Experimental Analysis of Behavior, 35, 227242.

Lee, V. L., \& Pegler, A. M. (1982). Effects on spelling of training children to read. Journal of Experimental Analysis of Behavior, 37, 311-322.

Litrownick, A. J., McInnis, E. T., Wetzel-Prichard, A. M., \& Felipelli, D. L. (1978). Restricted stimulus control and inferred attentional deficits in autistic and retarded children. Journal of Abnormal Psychology, 87, 554-562.

Lovaas, O. I., Koegel, R., \& Schreibman, L. (1979). Stimulus overselectivity in autism: A review of research. Psychological Bulletin, 86, 1236-1254.

Lovaas, O. I., Schreibman, L., Koegel, R., \& Rehm, R. (1971). Selective responding by autistic children to multiple sensory input. Journal of Abnormal Psychology, 77, 211-222.

Mackay, H. (1985). Stimulus equivalence in rudimentar reading and spelling. Analysis and Intervention in Developmental Disabilities, 5, 373-387.

Mackay, H. A., \& Sidman, M. (1984). Teaching new behavior via equivalence relations. In P. H. Brooks, 
R. Sperber, \& C. McCauley (Orgs.), Learning and cognition in the mentally retarded (pp.493-513). Hillsdale, N.J: Erlbaum.

Oliveira-Castro, J. M., Coelho D. S., \& Oliveira-Castro, G. A. (1999). Decrease of precurrent behavior as training increases: Effects of task complexity. The Psychological Record, 51, 343-355.

Rosa Filho, A. B., de Souza, D. G., de Rose, J. C. C., \& Hanna, E. S. (1999). Aprendendo a ler e escrever em pequenos passos. Software para pesquisa.

Saunders, R. R., \& Green, G. (1992). The nonequivalence of behavioral and mathematical equivalence. Journal of the Experimental Analysis of Behavior, 57, 227-241.

Saunders, R. R., Wachter, J., \& Spradlin, J. E. (1988). Establishing auditory stimulus control over an eightmember equivalence class via conditional discrimination procedures. Journal of the Experimental Analysis of Behavior, 49, 95-115.

Sidman, M. (1971). Reading and auditory-visual equivalences. Journal of Speech and Hearing Research, 14, 5-13.

Sidman, M. (1994). Equivalence relations: A research history. Boston, MA: Authors Cooperative.

Sidman, M., \& Tailby, W. (1982). Conditional discrimination vs. matching-to-sample: an expansion of the testing paradigm. Journal of the Experimental Analysis of Behavior, 37, 5-22.

Sidman, M., Willson-Morris, M., \& Kirk, B. (1986). Matching-to-sample procedures and the development of equivalence relations: The role of naming. Analysis and Intervention in Developmental
Disabilities, 6, 1-19.

Spradlin, J. E., \& Saunders, R. R. (1984). Behaving appropriately in new situations - A stimulus class analysis. American Journal of Mental Deficiency, 88, 574-579.

Stevens, K. B., Blackhurst, E., \& Slaton, D. B. (1991). Teaching memorized spelling with a microcomputer: Time delay and computer-assisted instruction. Journal of Applied Behavior Analysis, 24, 153-160.

Stoddard, L. T., \& Sidman, M. (1967). The effects of errors on children's performance on a circle-elipse discrimination. Journal of the Experimental Analysis of Behavior, 10, 261-270.

Stromer, R., McIlvane, W. J., Dube, W. V., \& Mackay, H. A. (1993). Assessing control by elements of complex stimuli in delayed matching to sample. Journal of Experimental Analysis of Behavior, 59, 83102.

Terrace, H. S. (1963a). Discrimination with and without errors. Journal of the Experimental Analysis of Behavior, 6,15-27.

Terrace, H. S. (1963b). Errorless transfer of a discrimination across two continua. Journal of the Experimental Analysis of Behavior, 6, 223-232.

Wilkinson, K. M., \& McIlvane, W. J. (2001). Methods for studying symbolic behavior and category formation: contributions of stimulus equivalence research. Developmental Review, 21, 355-374.

Submetido em 17 de abril de 2007 Aceito em 20 de janeiro de 2008 


\title{
PROGRAMAS DE PÓS-GRADUAÇÃO STRICTO SENSU EM ANÁLISE DO COMPORTAMENTO NO BRASIL BRAZILIAN GRADUATE PROGRAMS IN BEHAVIOR ANALYSIS
}

\author{
PROGRAMA DE PÓS-GRADUAÇÃO EM PSICOLOGIA \\ Centro de Educação e Ciências Humanas \\ Universidade Federal de São Carlos \\ Cursos: MESTRADO E DOUTORADO \\ Área de concentração \\ Comportamento e Cognição \\ Linhas de Pesquisa \\ 1. Análise comportamental da cogniçãoo \\ 2. Comportamento social e processos cognitivos
}

\section{DOCENTES:}

Almir Del Prette

Antonio Celso de Noronha Goyos

Azair Liane Mattos do Canto de Souza

Camila Domeniconi

Deisy das Graças de Souza

Elizabeth Joan Barham

Júlio César Coelho de Rose

Lúcia Cavalcanti de Albuquerque Williams

Maria Stella Coutinho de Alcântara Gil

Rosemeire Aparecida Scopinho

Susi Lippi Marques Oliveira

Zilda Aparecida Pereira Del Prette

Informações adicionais na página da internet: www.ppgpsi.ufscar.br

E-mail:ppgpsi@power.ufscar.br 